\title{
Assessment of the pertinence of infrared thermography as a diagnostic tool in sinusitis - Cases study
}

\author{
by E. Gauthier*, T. Marin*${ }^{\dagger}$, J-L. Bodnar and L. Stubbe**
}

* Département Recherche de l'École Supérieure d'Ostéopathie (ESO) Paris 8, rue Alfred Nobel - cité

Descartes, 77420, Champs-sur-Marne, France, laboratoire.descartes@eso-suposteo.fr

** GRESPI/Caractérisation Thermo-physique UFR Sciences Exactes et Naturelles.

Campus du Moulin de la Housse - BP 103951687 REIMS Cedex 2, France

${ }^{\dagger}$ Corresponding author: Thibault MARIN, Département Recherche, ESO Paris. thibault.marin@eso-suposteo.fr

\section{Abstract}

Sinusitis is a frequent otorhinolaryngologic disease, often misdiagnosed. None of the usual imaging equipment is specific, quick or efficient enough for sinusitis diagnosis. Misdiagnosis induces unnecessary health expenditures as well as a risk of antibiotic resistant strain development. Our study (with one healthy control subject, two pathological subjects) shows that infrared thermography could be an efficient, low-cost and non-invasive diagnostic tool for clinical purposes. A cohort study is necessary to establish threshold values for systematic reliable diagnosis.

\section{Introduction}

Sinusitis is an otorhinolaryngologic disease that affects people of every age, with millions of consultations per year [1]; it is fifth in ranking for antibiotics prescription [2,3]. Sinusitis is an inflammation of the mucous membrane of one or several of the sinus cavities of the face, sometimes accompanied by suppuration. It is a common affliction, although its prevalence is difficult to assess; according to an american study, it affects $14 \%$ of the American population, adults and children combined [4].

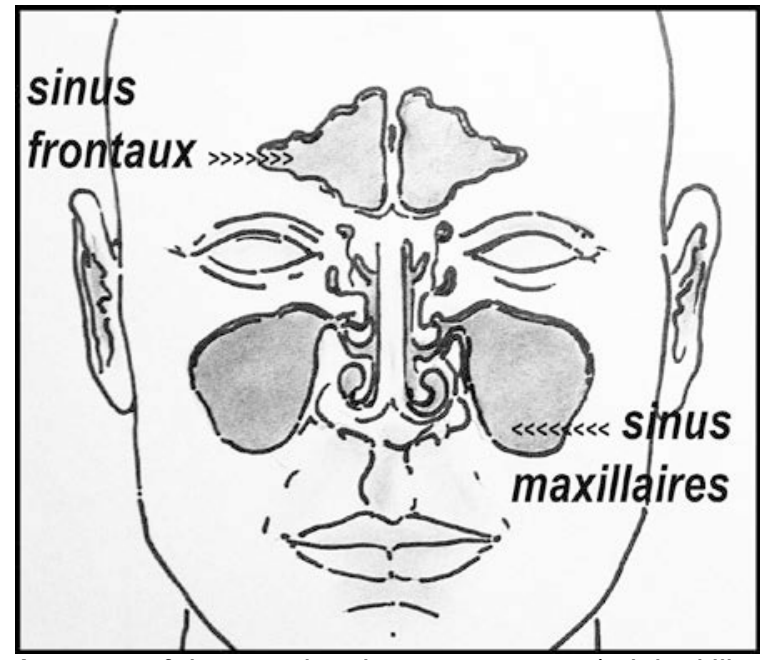

Fig.1. Anatomy of the anterior sinuses structure (original illustration)

The diagnosis is usually based on clinical examination conducted by a general practitioner; however, most symptoms of sinusitis are non-specific $[5,6]$. Studies show that half of the medical diagnosis of sinusitis based on clinical examination are false positives [7]. On that matter, sinusitis is a vast public health issue, because of diagnostic imprecision as well as inappropriate prescriptions. Medication - antibiotics - is the common medical treatment, regardless of the sinusitis etiology [8]. Sinusitis and its treatment raise issues: a financial issue due to medication costs and health expenditures related to sinusitis; a bacteriologic ecological issue due to the risk of antibiotic resistant strain [4]. In case of unsuccessful antibiotherapy, complication, surgical prevention or evolution to chronicity [9], many additional tests can be used: radiography [10], CAT scan [11], ultrasonography [12,13] and more [14,15]. To this day, none of these imagery tests constitutes a quick, noninvasive and efficient diagnostic tool for sinusitis

In this pathology, inflammation is materialized by a sinus temperature increase [16]. In humans, a calorific exchange happens between the skin and the atmosphere through infrared, either emitted or absorbed. Previous studies have shown that human inflammation can be detected through thermography: infrared cameras allow for a non-invasive estimation of infrared radiation, which are the external manifestation of an internal process $[17,18]$. 
The objective of this study is to assess the pertinence of infrared thermography as an efficient diagnostic tool for sinusitis. This study will only evaluate the anterior sinuses network (figure 1): it is less deep than the other sinuses of the face and thus more pertinent for thermographic evaluation. Also, 90\% of sinusitis affect the anterior sinuses network [19].

\section{Methods}

\subsection{Eligibility criteria}

Many pathologies, treatments, medications, aliments and behaviors can influence thermoregulation and/or infrared cutaneous radiation. In order to prevent interferences and measurement biases, the following parameters are non-inclusion criteria to this study:

- $\quad$ elderly people (over 70 years old) whose thermoregulation can be dysfunctional [20];

- consumption of alcohol, tea or coffee in the two hours before the study, the first one being a vasodilator, and the others being vasoconstrictors;

- local application of cream, balm or any other cutaneous treatment in the four hours before the study to avoid potent disruptions of the cutaneous infrared radiation;

- $\quad$ application of any manual therapy (massage, osteopathy, chiropractic, etc.) or any other kind of physiotherapy (electrotherapy, ultrasound, thermotherapy, hydrotherapy, etc.) during the six hours before the study, for the same reason;

- $\quad$ presence of a moustache and/or beard, for the same reason;

- consumption of veinotonics, vasoconstrictors and/or vasodilators (particularly antihypertensive medication) because of their thermoregulative pharmacokinetic;

- $\quad$ any endogenous thermal disruption (high or low-grade fever) or exogenous (physical effort, UV, etc.) during the four hours before the study;

- $\quad$ any head trauma in the last four weeks.

\subsection{Endpoints and objectivation tool}

This study endpoint is infrared cutaneous radiation of the face. According to the scientific literature, human skin emissivity is 0.98 [21], optimal length wave is between 7 and $10 \mu \mathrm{m}$ [22]; mean human skin temperature is $33.5^{\circ} \mathrm{C}$ [23], the thermal variation phenomenon is slow: for all these reasons, we prefer using a "long waves" bolometers matrix camera, which is simpler to implement and less expensive.

We use an infrared camera FLIR® $A 320$; its range of temperature measurement is between $-15^{\circ} \mathrm{C}$ and $250^{\circ} \mathrm{C}$, with a sensitivity of $0.07^{\circ} \mathrm{C}$ and an accuracy of $\pm 2^{\circ} \mathrm{C}$. The measurable lengthwave spectrum is between 7.5 and $13 \mu \mathrm{m}$. The lens field of view is $25^{\circ}$ with autofocus, image frequency is $30 \mathrm{~Hz}$, resolution is $320 \times 240$ pixels. The data acquisition is numeric through Ethernet connection, using the software FLIR $®$-IR Monitor. For this experiment, the camera settings are as follow: skin emissivity is 0.98 ; temperature level is $33.5^{\circ} \mathrm{C}$; temperature range of motion is $28.5^{\circ} \mathrm{C}$ to $40^{\circ} \mathrm{C}$.

\subsection{Study design}

This study design complies with the common design of medical researches using infrared thermography $[17,24-$ $26,32]$. It has been validated by the Commission of Evaluation and Selection of the Research Department of the ESO.

\subsubsection{Investigators}

A single investigator leads this experiment. He welcomes the subject, explains the research goal and the experimental protocol. He operates the camera and the software. The results analysis and synthesis are done by another investigator.

\subsubsection{Experimental protocol}

This experiment takes place in a dedicated room of the Research Department of the ESO; its volume is $72.5 \mathrm{~m}^{3}$, an important volume allows better thermal stability. For the same reason, air renewal is controlled to be as low as can be. Temperature is monitored to be constant at $22 \pm 1^{\circ} \mathrm{C}$, as is hygrometry at $45 \pm 5 \%$, in order to ensure subject's comfort as well as to minimize variations that can influence skin temperature [27]. The subject is sitting. The infrared camera is installed on a studio photographic tripod to ensure reproducibility of the thermograms acquisition. The camera lens is perpendicular to the frontal plan of the subject's face, in order to maximize collection of skin isotropic emission. The distance between the camera lens and the face is constant at $\approx 20 \mathrm{~cm}$, as a small distance increases the atmospheric transmission rate т [28]. To prevent any thermal or electromagnetic disruption, the camera setup is far of any potential disruptive element (air conditioning, doors, windows, computer station). The camera is turned on at least 30 minutes before any acquisition, in order to ensure thermal balance with the environment. 
The experimental protocol is explained to the subject, who signs an informed consent form. The patient then washes his face to prevent disruptions of the cutaneous infrared radiation. After an eight minutes passive waiting period - necessary for subject's temperature normalization [29] - the thermogram of the subject's face is recorded.

\subsection{Data analysis protocol}

As a first step, we assess each thermogram by a simple visual observation: the observation is systematic, and verifies each of the following elements:

- comparative evaluation of global frontal area and global maxillary area;

- comparative evaluation of left frontal area and right frontal area;

- comparative evaluation of left maxillary area and right maxillary area;

Secondly, for quantitative comparison, we measure average temperatures of each of the four sinuses areas: left Frontal sinus (LF), right Frontal sinus (RF), left Maxillary sinus (LM), right Maxillary sinus (RM). For this purpose, we use the software FLIR® ThermaCAM Researcher Professional 2.8 SR-1. Each area is a circular surface set manually using known anatomical and radiological data (figure 2); the accuracy of the software is of $0.1^{\circ} \mathrm{C}$.

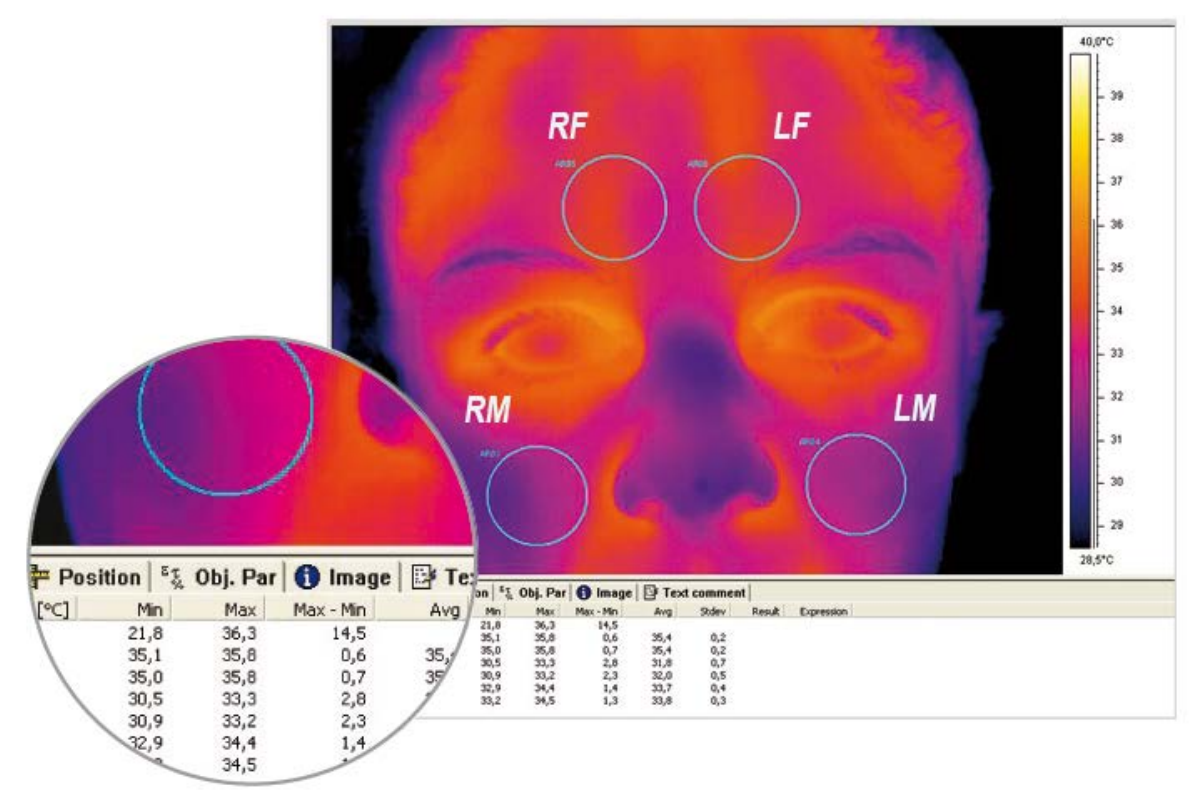

Fig.2. setup for quantitative analysis with FLIR® ThermaCAM Researcher Professional 2.8 SR-1

For each thermogram, the analysis goes as follow:

- comparison of global frontal and maxillary average temperatures;

- comparison of left and right frontal average temperatures;

- comparison of left and right maxillary average temperatures.

\section{Results}

\subsection{Subjects description}

Subject \#1 is a 27 years old woman, $1.69 \mathrm{~m} 63 \mathrm{~kg}(\mathrm{IMC}=22.1)$; she is a healthy control patient. Subject \#2 is a 22 years old woman, $1.72 \mathrm{~m} 52 \mathrm{~kg}$ (IMC=17.6); she suffers from bilateral maxillary sinusitis. Subject \#3 is a 23 years old woman, $1.64 \mathrm{~m} 60 \mathrm{~kg}(\mathrm{IMC}=22.3)$; she suffers from unilateral left frontal sinusitis.

\subsection{Data analysis}

Visual assessment of subject \#1 thermogram (figure 3) draws us to two important observations: first, an important temperature difference exists between the frontal area and the maxillary area that is colder; second, symmetry between the left and right halves of the face is observed.

Visual assessment of subject \#2 thermogram (figure 4) shows a difference between left and right frontal areas: a lighter vertical shaped spot can be observed on the right frontal area. The visual aspect of maxillary areas is marbled, warmer surfaces of variable size and shape are observable with two lighter spots in the right area and one in the left 
area. The difference observed on figure 3 between the warmer frontal areas and the colder maxillary areas is verified here.

Visual assessment of subject \#3 thermogram (figure 5) shows an asymmetry between the left and right frontal areas, the left area being much lighter, thus much warmer. No difference is observed between left and right maxillary areas. The difference observed on figure 3 between the warmer frontal areas and the colder maxillary areas is verified here.

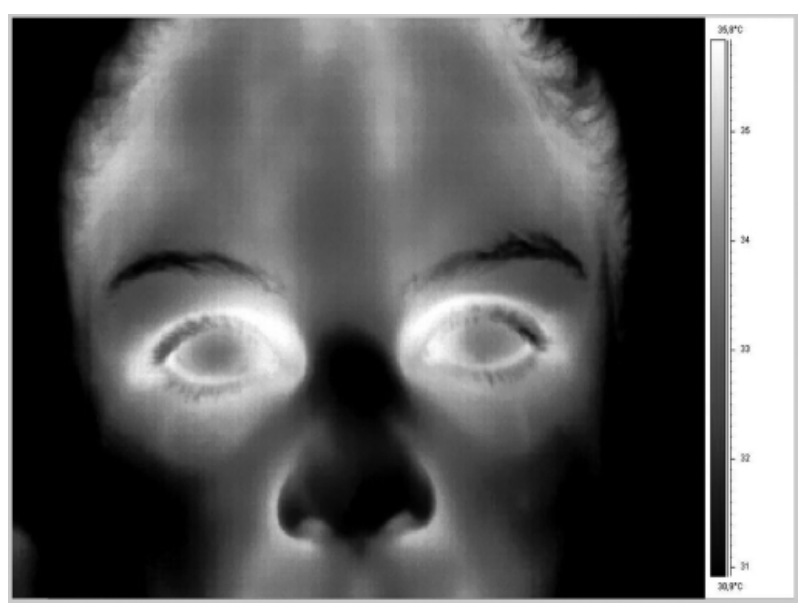

Fig. 3. Subject \#1, healthy control patient. Contrasted thermogram.

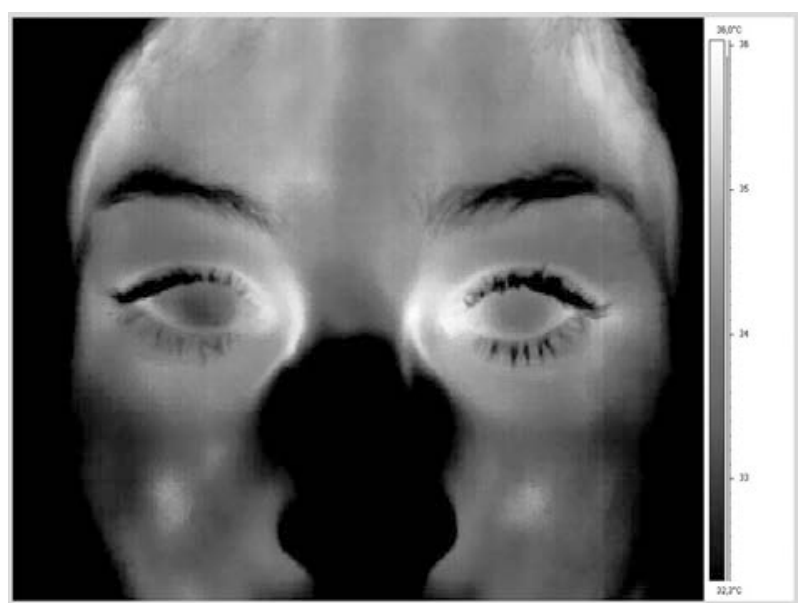

Fig. 4. Subject \#2, bilateral maxillary sinusitis. Contrasted thermogram.

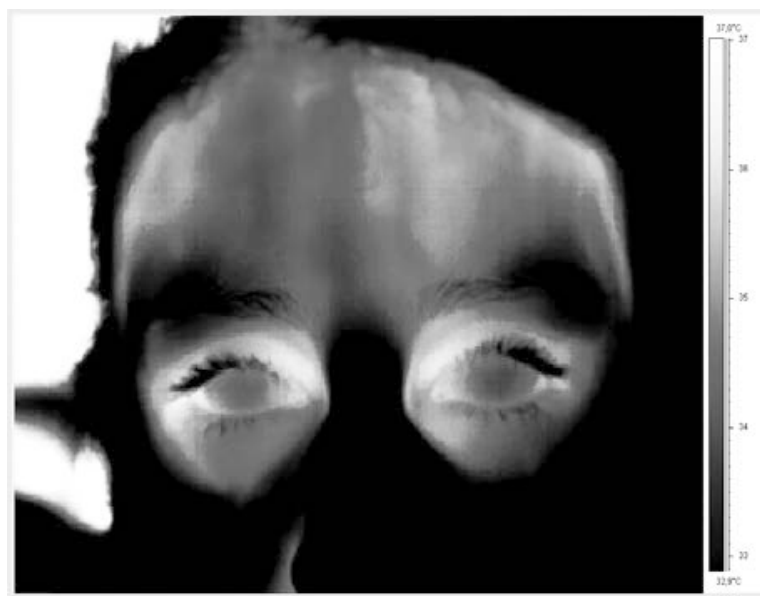

Fig..5. Subject \#3, unilateral left frontal sinusitis. Contrasted thermogram. 
Measured data is summarized in table 1. The differences between average global frontal temperature and average global maxillary temperature (table 1) confirms our visual assessment: the frontal area is consistently warmer than the maxillary area. For our healthy control subject, this difference is close to $2^{\circ} \mathrm{C}$. The temperature difference between frontal and maxillary areas is easily explained by the presence of subcutaneous adipose tissue in the latter, constituting an insulating layer [30].

We then calculate the differences between left and right frontal and maxillary areas (table 2). For our healthy subject, a very small difference of $0.2^{\circ} \mathrm{C}$ is found in both cases. Subject \#3 suffers from unilateral left frontal sinusitis: our measurements show that the left frontal sinus is indeed warmer $\left(+0.7^{\circ} \mathrm{C}\right)$; no difference is found in maxillary areas. For subject \#2, the difference between left and right maxillary areas $\left(+0.2^{\circ} \mathrm{C}\right)$ is very small - similar to the healthy subject measurements. This is normal, as she suffers from bilateral maxillary sinusitis. The right frontal area is slightly warmer $\left(+0.4^{\circ} \mathrm{C}\right)$, as observed visually: this leads us to suspect a possible right frontal sinusitis.

Table 1 - Average sinuses temperature (in $\left.{ }^{\circ} \mathrm{C}\right)$; average frontal (avgF) and maxillary (avgM) areas, and Frontal/Maxillar differential

\begin{tabular}{llllllll}
\hline Subject & LF & RF & LM & RM & avgF & avgM & avgF - avgM \\
\hline$\# 1$ & $33.8 \pm 0.3$ & $33.7 \pm 0.4$ & $32.0 \pm 0.5$ & $31.8 \pm 0.7$ & 33.8 & 31.9 & 1.9 \\
$\# 2$ & $34.4 \pm 0.1$ & $34.8 \pm 0.1$ & $34.0 \pm 0.3$ & $33.8 \pm 0.4$ & 34.6 & 33.9 & 0.7 \\
$\# 3$ & $34.9 \pm 0.2$ & $34.2 \pm 0.2$ & $31.2 \pm 0.6$ & $31.2 \pm 1.0$ & 34.6 & 31.2 & $3.4 f$ \\
\hline
\end{tabular}

Table 2 - Left/right measurements comparison (in ${ }^{\circ} \mathrm{C}$ )

\begin{tabular}{llllllll}
\hline Subject & $\mathbf{F g}$ & $\mathbf{F d}$ & $\mathbf{\Delta} \mathbf{F}$ & $\mathbf{M g}$ & $\mathbf{M d}$ & $\mathbf{\Delta M}$ & $\mathbf{\Delta M}$ \\
\hline$\# 1$ & 33.8 & 33.7 & +0.1 & 32.0 & 31.8 & +0.2 & $31.9 \pm 0.6$ \\
$\# 2$ & 34.4 & 34.8 & -0.4 & 34.0 & 33.8 & +0.2 & $33.9 \pm 0.4$ \\
$\# 3$ & 34.9 & 34.2 & +0.7 & 31.2 & 31.2 & 0.0 & $31.2 \pm 0.8$ \\
\hline
\end{tabular}

\section{Discussion}

Visual analysis is fast, and consistent with the medical diagnosis in both pathological cases of this study. A potential false positive is observed on one subject, but could also be a true positive of an asymptomatic frontal sinusitis or misdiagnosed by the previous medical examination. For this reason, it is important to cross the results with the clinical symptoms (facial pain, rhinorrheas), in order to save time and prevent misdiagnosis that lead to expensive and inefficient courses of treatment.

The quantitative analysis is consistent with the visual analysis. However, our analysis protocol has reached a limit: we have no way of differentiating a normal temperature with a bilateral sinusitis, since the difference between left and right is rightfully not significant. In order to be able to diagnose bilateral pathologies, a different analysis is required, using a reference temperature to which can be compared each measurement. A pertinent anatomical element would be the medial canthi, since several previous studies have shown that it's the closest to the core body temperature [31,32]. A larger study with a group of healthy control subjects in order to establish thershold values, and a group of pathological subjects to verify on a wider scale the pertinence and efficiency of infrared thermography is required.

\section{Conclusion}

Infrared thermography seems to be an efficient, reliable, non-invasive and immediate diagnostic tool for sinusitis; it could be used with any patient, including children, pregnant women or immunodepressed people. Many thermal cameras are light, easy to use and inexpensive (under 1000€). For research purposes, a wider study is required to establish threshold values to precise the analysis protocol. Visual analysis crossed with clinical symptoms could be sufficient for clinical diagnosis. 


\section{REFERENCES}

[1] Benson V, Marano MA. Current estimates from the National Health Interview Survey. 1995. Vital Health Stat. 1998;199:1-428.

[2] Fagnan LJ. Acute sinusitis: a cost-effective approach to diagnosis and treatment. Am Fam Physician. 1998;58:1795-802,805-6.

[3] McCaig LF, Hughes JM. Trends in antimicrobial drug prescribing among office-based physicians in the Unites States. JAMA. 1995;273:214-9.

[4] Poole MD. A focus on acute sinusitis in adults: changes in disease management. Am J Med. 1999;106:38S-47S.

[5] Lacroix JS, Richetti A, Lew D et al. Symptoms and clinical and radiological signs predicting the presence of pathogenic bacteria in acute rhinosinusitis. Acta Otolaryngol. 2002;122:192-6.

[6] Shields G, Seikaly H, Le Boeuf M et coll. Correlation between facial pain or headache and computed tomography in rhinosinusitis in Canadian and U.S. Subjects. Laryngoscope. 2003;113:943-5.

[7] Dewey CS, Robert MH. Acute bacterial rhinosinusitis in adults: part I. Evaluation. Am Fam Physician 2004;70:1685-91.

[8] Kaiser L. Du bon et mauvais usage des antibiotiques dans les sinusites. Forum Médical Suisse. 2003;36:843-7.

[9] Barry B. Imagerie pour une sinusite : radiologie standard, scanner ou IRM ? Correspondance en médecine générale. 2001;1vol2:49-50.

[10] Skinner DW, Richards SH. A comparison between sinus radiographic findings and the macroscopic appearances of the paranasal sinus mucosa. Ear Nose Throat J. 1991;70:169-72.

[11] Gwaltney JM Jr, Phillips CD, Miller RD, Riker DK. Computed tomographic study of the common cold. N Engl J Med. 1994;330:25-30.

[12] Laine K, Maatta T, Varonen H, Makela M. Diagnosing acute maxillary sinusitis in primary care : a comparison of ultrasound, clinical examination and radiography. Rhinology 1998;36(1):2-6.

[13] Shapiro GG, Furukawa CT, Plerson WE et al. Blinded comparison of maxillary sinus radiography and ultrasound for diagnosis of sinusitis. J Allergy Clin Immunol 1986; 77: 59-64.

[14] International Rhinosinusitis Advisory Board. Infectious rhinosinusitis in adults:classification, etiology and management. Ear Nose Throat J 1997;76(12suppl):1-22.

[15] Le AV,Simon RA.Making the call:the diagnosis of acute community- acquired bacterial sinusitis. Am J Rhinol. 2006;20 (6):658-61.

[16] Prasał M, Sawicka KM, Wysokiński A. Thermography in cardiology. Kardiol Pol. 2010;68(9):1052-6.

[17] Ring EFJ, Ammer K. Infrared thermal imaging in medicine. Physiological Measurement. 2012;33(3):R33.

[18] Lange KHW, Jansen T, Asghar S, Kristensen PL, Skjønnemand M, Nørgaard P. Skin temperature measured by infrared thermography after specific ultrasound-guided blocking of the musculocutaneous, radial, ulnar, and median nerves in the upper extremity. British journal of anaesthesia. 2011;106(6):887-95.

[19] Institut Français de Chirurgie du Nez et des Sinus. http://www.institut-nez.fr/sinusite/sinusite-chronique-19.html. Dernière mise à jour le 26/04/2011.

[20] Khan F, Belch JJ. Cutaneous vascular responses and thermoregulation on relation to age. Clinical Science. 1992;85(5):521-8.

[21] Steketee J. Spectral emissivity of skin and pericardium. Physics in Medicine and Biology. 1973;18(5):686-94.

[22] Bowling Barnes R. Infrared-radiant energy provides new concepts and instrumentation for medical diagnosis. Science. 1963;870-7.

[23] Freitas RA Jr. Nanomedicine, volume I: Basic capabilities. Austin TX : Landes Bioscience ; 1999. 509p.

[24] Head JF, Elliott RL. Infrared imaging: making progress in fulfilling its medical promise. IEEE Eng Med Biol Mag. 2002;21:80-5.

[25] Buzug TM, Schimann S, Pfaffmann L, Reinhold U, Ruhlmann J. Functional infrared imaging for skin-cancer screening. Cong Proc IEEE Eng Med Biol Soc. 2006;1:2766-9.

[26] Bharara M, Cobb JE, Claremont DJ. Thermography and thermometry in the assessment of diabetic neuropathic foot: a case for furthering the role of thermal techniques. Int J Low Extrem Wounds. 2006;5:250-60.

[27] Ming Z, Zaproudina N, Siivola J, Nousiainen U, Pietikainen S. Sympathetic pathology evidenced by hand thermal anomalies in carpal tunnel syndrome. Pathophysiology. 2005;12(2):137-41.

[28] Institut Wallon ASBL. La thermographie infrarouge en maintenance prédictive, cas du centre hospitalier SaintJoseph-Espérance. Jambes (Belgique) : Ministère de la région Wallone, DGTRE, Service de l'énergie.

[29] Roy R, Boucher JP, Comtois AS. Digitized Infrared Segmental Thermometry: time requirements for stable recordings. Journal of Manipulative and Physiological Therapeutics 29(6):2006;468e1-468e10.

[30] Savastano DM, Gorbach AM, Eden HS, Brady SM, Reynolds JC, Yanovski JA. Adiposity and human regional body temperature. The American journal of clinical nutrition. 2009;90(5):1124-31.

[31] Ng EY, Kaw GJ, Chang WM. Analysis of IR thermal images for mass blind fever screening. 2004;68(2):104-9.

[32] Ring EFJ, Jung A, Zuber J, Rutstecka A, Kalicki B, Vardasca R. Medical infrared imaging: principles and practices,1st ed. New York, CRC Press;2012.638p. 